\title{
Tecnología positiva. El uso de la tecnología para mejorar el bien- estar personal y las interacciones sociales
}

María José Distéfano ${ }^{1}$, Jaime O'Conor ${ }^{2}$, María Celina Mongelo ${ }^{3}$ y María Cristina Lamas ${ }^{4}$

\section{Artículo}

Material original autorizado para la publicación en la revista Psicodebate. Facultad de Ciencias Sociales. Universidad de Palermo.

Recibido 08-01-2015 | Aceptado 01-06-2015

\section{Resumen}

La Tecnología Positiva es un nuevo campo que combina los avances tecnológicos con los aportes de la Psicología Positiva. Esta última ilumina el modo en que las TICs pueden aplicarse para fomentar las emociones positivas, promover el crecimiento personal y contribuir al desarrollo social. La Tecnología Positiva se propone mejorar las características de las experiencias personales con el objetivo de ampliar el bienestar y fomentar el desarrollo de fortalezas en sujetos, organizaciones y sociedades. En este trabajo se describe brevemente la teoría de la Felicidad de Martin Seligman y se realiza una revisión teórica de la literatura existente sobre Tecnología Positiva, identificando sus características y objetivos fundamentales así como también los desarrollos más recientes de esta emergente disciplina.

Palabras Clave: tecnología positiva, psicología positiva, bienestar, tecnologías de información y comunicación, intervenciones online.

1 PsicoTech, Universidad Católica Argentina - Argentina; mjdistefano@uca.edu.ar

2 PsicoTech, Universidad Católica Argentina - Argentina.

3 PsicoTech, Universidad Católica Argentina - Argentina.

4 PsicoTech, Universidad Católica Argentina - Argentina. 


\section{Abstract}

Positive technology is a new field that combines the new developments in technology with the contributions of Positive Psychology. The latter guides the way in which ICTs can be used to foster positive emotions, promote personal growth and contribute to social development. Positive Technology intends to improve personal experiences so as to enhance the well-being of human beings and promote the nurturing of strengths in people, organizations and societies. This study briefly describes Martin Seligman's Happiness Theory and aims at reviewing the state-of-art of Positive Technology by identifying its essential characteristics and objectives, as well as its most recent developments.

Keywords: positive technology, positive psychology, well-being, icts, internet-based interventions, ICTs. 
La sociedad actual, caracterizada por la globalización, la complejidad, la diversidad y la virtualidad, ha naturalizado los desarrollos de la tecnología que atraviesa la vida cotidiana de los sujetos. Diversos estudios señalan los perjuicios y las consecuencias negativas de su abuso o mal uso. Sin embargo, existen también desarrollos que aprovechan los beneficios de su aplicación, entre ellos algunos en el área de la salud.

La incorporación de la Tecnología en la Clínica Psicológica, si bien es poco conocida en Argentina, lleva ya más de dos décadas. La Psicoterapia Asistida por Computadoras incluye diversos dispositivos con múltiples fines, ya sea de comunicación, evaluación o complemento terapéutico.

En los últimos años, y dentro el marco del enfoque salugénico, se ha desarrollado un nuevo campo: la Tecnología Positiva, que estudia el impacto de las tecnologías en el crecimiento personal de los individuos y el desarrollo social.

En el presente trabajo se describe brevemente la teoría de la Felicidad de Martin Seligman y se realiza una revisión teórica de la literatura existente sobre la Tecnología Positiva, identificando sus características y objetivos fundamentales así como también sus desarrollos más recientes.

\section{Psicología Positiva}

El surgimiento del enfoque salugénico en psicología se ubica en la década de los 80 del siglo pasado. El mismo se centra en el concepto de promoción de la salud, complementando al modelo médico centrado en la enfermedad. Años más tarde, en 1998, la Psicología Positiva (PP) comienza a cristalizarse cuando el Dr. Martin Seligman asume como presidente de la Asociación Americana de Psicología y propone como principal interés el bienestar y todo lo que puede potenciarse para estar aún mejor (Gancedo, 2008).

La PP no se trata de un movimiento filosófico ni espiritual, ni son ejercicios de autoayuda para alcanzar la felicidad. Según Castro Solano (2010), tampoco se trata de la negación del sufrimiento o aspectos negativos de los sujetos, sino más bien de tender hacia la complementariedad de enfoques y recuperar el equilibrio que la psicología perdió en los últimos sesenta años al otorgarle mayor énfasis a los aspectos patológicos de las personas. Es una nueva disciplina que tiene por objeto de estudio el funcionamiento psíquico óptimo. Se reconocen entre sus antecedentes los desarrollos de autores como Rogers y Maslow. Su objetivo principal se focaliza en las fortalezas y virtudes de los individuos para potenciarlas hacia el florecimiento individual, comunitario y social. Al decir de su pionero, Seligman (2002), el principal objetivo de la psicología positiva es comprender y facilitar la felicidad y el bienestar subjetivo; potenciar los rasgos positivos del 
individuo, cultivar sus fortalezas y promover el cambio positivo. El foco está puesto en lo que sí funciona en la vida de las personas, en el estudio científico de las experiencias subjetivas positivas, los rasgos individuales positivos, las instituciones que facilitan su desarrollo y los programas que ayudan a mejorar la calidad de vida de los individuos, lo que a su vez redunda en la prevención de los trastornos psicopatológicos (Seligman \& Csikszentmihalyi, 2000).

En el año 2002, Seligman presentó su teoría de la Auténtica Felicidad (AF), en la que sostenía que el estudio de la felicidad constituía el propósito general de la PP considerando tres rutas o vías de acceso a la misma: la vida placentera, la vida comprometida y la vida con sentido. Debe resaltarse que estas tres vías son complementarias. Se puede alcanzar la vida plena por cualquiera de ellas, pero quienes emplean las tres rutas conjuntamente experimentarán mayor satisfacción (Peterson, 2006).

La vida placentera es aquella que se obtiene a través de la experiencia de emociones positivas y es la más conocida. Corresponde a la propuesta hedonista en la que se busca incrementar la cantidad de emociones positivas la mayor parte del tiempo. Entre los beneficios de las emociones positivas se destaca que, según Fredrickson (1998), amplían los repertorios de pensamiento y acción, construyendo así recursos personales duraderos. Los recursos para alcanzar esos momentos felices a lo largo de la vida son el savoring (saboreo) y el mindfulness (conciencia plena), cuando se persigue rescatar el momento presente de la forma más genuina. Consiste en percibir, apreciar y disfrutar los sentidos de forma auténtica, y en alcanzar un estado mental que mantiene la atención plena en los propios pensamientos, acciones o sensaciones. Cuando se trata de focalizar en el pasado, las emociones positivas se obtienen al cultivar la gratitud y el perdón, al agradecer las experiencias vividas y al darle un nuevo sentido a experiencias dolorosas pasadas, transformando afectos, pensamientos y acciones hacia el sujeto de dicha experiencia. En la proyección al futuro también pueden experimentarse emociones positivas, a través de la esperanza y el optimismo. Se basa en la habilidad para generar caminos alternativos frente a las dificultades, adoptando un estilo atribucional inestable, específico y externo para explicar las causas de los fracasos, y otro interno, global y estable para los logros.

La vida placentera tiene dos límites. Por un lado, Lyubomirsky (2008) sostiene que el nivel de emocionalidad positiva es en gran parte fijo ya que depende un $50 \%$ de dos factores estables: la personalidad y los componentes genéticos de cada individuo. Por otro lado, es fácil habituarse a las emociones positivas por lo que resulta necesario incrementarlas cada vez más hasta el punto en que no causan mayor felicidad. Esto se denomina habituación o adaptación hedónica.

En este nivel se alcanza el llamado bienestar subjetivo o emocional, es decir, la evaluación personal de la satisfacción con la propia vida y de sus emociones 
positivas o negativas en distintos dominios de satisfacción, como ser el trabajo, la familia, el ocio, la salud y los ingresos (Diener, 1994).

Por su parte, la vida comprometida implica el desarrollo y crecimiento personal. En este nivel el bienestar se asocia con conductas dirigidas al autodesarrollo o realización personal, y la experiencia de bienestar procede del compromiso con la actividad y con la capacidad de experimentar flow. Se alcanza el estado de flow (Csikszentmihalyi, 1997) cuando una tarea absorbe al sujeto de modo tal que se pierde la noción del tiempo, y cuando hay una justa correlación entre las habilidades personales del individuo y las características propias de la tarea. Para ello es necesario conocer las propias fortalezas que, aplicándolas en una tarea concreta, permitirán alcanzar esa conexión particular llamada compromiso. En la experiencia de flow hay objetivos claros, retroalimentación inmediata, presencia de desafíos, habilidades propias del sujeto en juego, alto nivel de concentración, gran involucración, motivación intrínseca y emociones positivas. El flow no es objetivo, pues se vincula con la percepción subjetiva del desafío y las habilidades. Se denomina vida participativa o comprometida a esta vía, pues se relaciona con el bienestar eudaimónico, en el que la gratificación exige esfuerzo y trabajo, pero a la vez es más plena y duradera (Castro Solano, 2010).

La tercera vía, menos estudiada, se relaciona con la búsqueda de sentido, entendida en un contexto global amplio. Se incorpora en este nivel el aspecto social o interpersonal, y se alcanza por el servicio a propósitos que trascienden lo personal. Por eso a esta ruta conocida como la vida con sentido se la asocia con el bienestar social. Consiste en la aplicación de las fortalezas personales para el desarrollo de algo que trascienda la propia individualidad, al servicio de los demás, para colaborar con otro y fomentar el desarrollo de sus potencialidades (Seligman, 2002). El resultado de este proceso es el significado vital, que suele darse a través de las instituciones positivas tales como la familia, el trabajo o la escuela: el pertenecer y servir a algo considerado superior a sí mismo.

Años más tarde, Seligman (2011) hizo un giro desde la teoría de la AF a la Teoría del Bienestar (TB). Esto se debió a que la felicidad está socialmente ligada a experimentar emociones positivas, pero poco a la entrega y al sentido. La TB considera que el núcleo de la psicología positiva es el bienestar, entendido como un constructo compuesto por varios elementos operacionalizables y mensurables: emociones positivas, compromiso, relaciones positivas, sentido y logros. Aunque ninguno de estos cinco elementos define el bienestar por sí mismo, podrían considerarse los pilares de la plenitud y realización como seres humanos. Se los conoce por su sigla en inglés, PERMA. Esta no es una prescripción o fórmula exacta que se aplica a todos por igual, sino más bien una descripción de distintos factores que contribuyen al florecimiento humano. 


\section{Psicoterapia Asistida por Computadoras}

Los desarrollos de las Tecnologías de Información y Comunicación (TICs) se han incorporado al ámbito de la salud mental, abriendo nuevas perspectivas en los tratamientos psicoterapéuticos. La predicción que hace una década hicieron Norcross, Hedges, y Prochaska (2002), al señalar que entre las intervenciones terapéuticas de mayor crecimiento estarían aquellas caracterizadas por el uso de nuevas tecnologías, se ha confirmado. Aún más, dicha tendencia continuará su curso según la última versión del estudio Delphi publicada en 2013 (Norcross, Pfund, \& Prochaska, 2013). Los recursos tecnológicos no reemplazan la psicoterapia tradicional sino que ofrecen un complemento para optimizar los abordajes tradicionales y maximizar el alcance de los tratamientos a una mayor cantidad de personas (Bunge, López, Mandil, Gomar, \& Borgialli, 2009).

La Psicoterapia Asistida por Computadora (PAC) es definida de manera genérica como cualquier sistema informático que ayuda a los profesionales a diseñar y/o aplicar tratamientos psicoterapéuticos (Marks, Cavanagh, \& Gega, 2007). La psicoterapia se ha valido de las tecnologías en este sentido amplio desde hace ya bastante tiempo. En una etapa se les dio lugar con fines de evaluación, en otra con fines de comunicación (sincrónica y diacrónica), y actualmente se las utiliza como complemento del abordaje tradicional. Aquí es donde se encuentran los desarrollos de Realidad Virtual (RV) o Aumentada, así como también las aplicaciones de Biofeedback, el uso de los Juegos Serios (JS), y las aplicaciones online para distintos dispositivos como PC, Smartphones o tablets. Estas últimas son aplicaciones distribuidas por internet y autoadministrables que cumplen fines de promoción de la salud, prevención, o abordaje de ciertas patologías en un estadio leve. Están disponibles a toda hora y en cualquier lugar en que se cuente con un dispositivo con acceso a internet, lo cual permite llegar a muchas más personas que no acuden a buscar ayuda psicológica por diversos motivos, o que no llegan a ser atendidas por falta de recursos profesionales humanos (Botella, Quero, Serrano, Baños, \& García-Palacios, 2009; Mandil, Bunge, Gomar, Borgialli, \& Labourt, 2009). Cabe señalar que una vez desarrollados estos sistemas, los costos son bajos (de mantenimiento y actualización) con relación a los costos de abordaje uno a uno.

\section{Tecnología Positiva}

Existe la posibilidad de combinar los objetivos de la Psicología Positiva con los desarrollos de las TICs en un nuevo paradigma: la Tecnología Positiva (Botella et al., 2012b). Su principal propósito es utilizar la tecnología para 
mejorar las características de las experiencias personales con el objetivo de mejorar el bienestar, fomentar el desarrollo de fortalezas y resiliencia en sujetos, organizaciones y sociedades (Riva, 2012).

¿Cómo puede la tecnología contribuir al bienestar? La respuesta sólo se puede construir teniendo en cuenta la tecnología como herramienta y no como fin. Una cuestión es desarrollar tecnología y otra es su aplicabilidad. La psicología positiva puede sugerir cómo desarrollar sistemas y aplicaciones que fomenten emociones positivas, promuevan el crecimiento personal y desarrollen la creatividad, mientras contribuyen al desarrollo social y cultural. La Tecnología Positiva (TP) es definida como el abordaje científico y aplicado del uso de la tecnología para potenciar la calidad de nuestra experiencia personal mediante su estructuración, aumento y/o reemplazo (Botella et al., 2012b; Riva, 2012). Está orientada a diseñar sistemas que promuevan el empoderamiento y el bienestar en individuos y grupos de distintos niveles sociales, siendo su marco teórico la psicología positiva, y ha demostrado su capacidad de transformar productiva y positivamente la salud mental en poblaciones europeas y americanas (Wiederhold, 2012).

Si bien múltiples investigaciones han demostrado que las intervenciones en psicología positiva (IPP) son favorables para alcanzar emociones positivas y bienestar, según Drozd, Mork, Nielsen, Raeder, y Bjørkli (2014) muchas personas no desean consultar a un profesional para mejorar su bienestar. Asimismo, sostienen que la distribución de intervenciones orientadas a incrementar el bienestar por internet parece prometedora y con la misma eficacia que cara a cara. Por su parte, Rose (2014) sostiene que las BITs (Behavioral Intervention Technologies, aplicaciones para fomentar conductas que promueven la salud y el bienestar) en el marco de la PP pueden incrementar el entrenamiento en estrategias saludables comprobadas empíricamente prescindiendo de tener que entrenar a profesionales en esos abordajes. En este sentido, existen múltiples aplicaciones con intervenciones automatizadas y autoadministrables con diferentes objetivos (ver tabla 1). 
Tabla 1.

Intervenciones automatizadas y autoadministrables

\begin{tabular}{|c|c|c|}
\hline Aplicación & Referencia & Objetivos y Características \\
\hline $\begin{array}{l}\text { Sonreír es } \\
\text { Divertido }\end{array}$ & $\begin{array}{l}\text { Botella et al. } \\
\text { (2012a) }\end{array}$ & $\begin{array}{l}\text { Su objetivo es la prevención de síntomas de depre- } \\
\text { sión y ansiedad. } \\
\text { Promueve estrategias de regulación emocional; } \\
\text { evoca emociones positivas y desarrolla las propias } \\
\text { fortalezas. }\end{array}$ \\
\hline Live Happy & $\begin{array}{l}\text { Parks, Della Por- } \\
\text { ta, Pierce, Zilca, } \\
\text { y Lyubomirsky } \\
(2012)\end{array}$ & $\begin{array}{l}\text { Fomenta la felicidad mediante actividades como } \\
\text { ejercicios de gratitud, bondad, fomento de relacio- } \\
\text { nes personales, prosecución de metas, y reminis- } \\
\text { cencia de días felices. }\end{array}$ \\
\hline Better Days & $\begin{array}{l}\text { Drozd et al. } \\
(2014)\end{array}$ & $\begin{array}{l}\text { Cuenta con intervenciones con relación a la grati- } \\
\text { tud, actividades placenteras, fortalezas, actos de } \\
\text { bondad, optimismo, flow, procesos atribucionales } \\
\text { y mindfulness. }\end{array}$ \\
\hline $\begin{array}{l}\text { EARTH of Well- } \\
\text { being }\end{array}$ & $\begin{array}{l}\text { Baños et al. } \\
(2014) \\
\text { Baños, Botella, } \\
\text { Etchemendy y } \\
\text { Farfallini, (2012) }\end{array}$ & $\begin{array}{l}\text { Promueve el desarrollo de emociones positivas, el } \\
\text { entrenamiento en regulación emocional, y el poten- } \\
\text { ciamiento de distintas fortalezas de carácter. }\end{array}$ \\
\hline AB Intervention & $\begin{array}{l}\text { Martínez-Martí et } \\
\text { al. (2014) }\end{array}$ & $\begin{array}{l}\text { Consta de ejercicios destinados a incrementar la } \\
\text { conciencia de la belleza y de cómo ello afecta las } \\
\text { emociones, pensamientos y comportamientos. } \\
\text { Asimismo, busca cultivar una actitud estética frente } \\
\text { a la belleza en el arte, la naturaleza y la moralidad. }\end{array}$ \\
\hline InJoy & $\begin{array}{l}\text { Redzic et al. } \\
(2014)\end{array}$ & $\begin{array}{l}\text { Programa psicoeducacional para adolescentes. } \\
\text { Se enseñan habilidades para prevenir la depresión } \\
\text { y reducir síntomas primarios, diseñado para ser } \\
\text { utilizado en ámbitos escolares. }\end{array}$ \\
\hline SMART-OP & Rose (2014) & $\begin{array}{l}\text { Programa de intervenciones comportamentales } \\
\text { para el control de estrés (como la respiración } \\
\text { focalizada, relajación muscular guiada) y el entre- } \\
\text { namiento en resiliencia. Su foco es la capacitación } \\
\text { y el entrenamiento de habilidades de resiliencia no } \\
\text { en situaciones clínicas, pero sí sobre la base de la } \\
\text { posibilidad de utilizarlas al hacer frente a eventos } \\
\text { estresantes de todos los días. }\end{array}$ \\
\hline
\end{tabular}

Por lo general las aplicaciones incluyen una sola estrategia. Sin embargo se ha encontrado que los sujetos que buscan la felicidad online suelen preferir una variedad de actividades, lo que a su vez predice mayores mejorías (Baños et al., 2014). Existen estudios que comprueban la aceptación y efectividad de 
IPP ofrecidas como aplicaciones online con diferentes ejercicios. Asimismo, se comprobó que lo más conveniente es ofrecer entre dos y cuatro actividades diferentes, suficiente como para ofrecer variedad y efectos a largo plazo (Drozd et al., 2014; Schueller \& Parks, 2012).

Si bien los efectos son modestos, las intervenciones mediatizadas por internet proveen una buena opción rentable que puede ser adecuada como una intervención temprana y de baja intensidad. Martínez-Martí et al. (2014) sostienen que el formato online tiene distintas ventajas como ser que una vez que está desarrollada la intervención requiere poco esfuerzo mantenerla. A su vez, los impedimentos espaciotemporales son eliminados, ya que al estar disponibles online 7x24 se puede acceder a ellos desde cualquier lugar y a cualquier hora mientras se cuente con un dispositivo con conexión a internet (Rose, 2014). En este sentido, Morris y Picard (2014) sostienen que las BITs autoguiadas tienen la posibilidad de proliferarse ampliamente alcanzando poblaciones que de otra manera tal vez no podrían recibir ningún tipo de ayuda.

Como contrapartida, algunos sostienen que en las intervenciones computarizadas que son absolutamente autoadministrables sin la supervisión de un clínico o experto, disminuyen significativamente la adherencia y motivación (Christensen, Griffiths, \& Farrer, 2009). En este sentido, Redzic et al. (2014) señalan la importancia de encontrar la manera de mantener el compromiso del usuario para terminar el programa en abordajes de prevención e intervención temprana, lo cual puede ser difícil por la poca motivación ante la ausencia de sintomatología. Sugieren ofrecer aplicaciones que sean agradables, atractivas y entretenidas para una población tanto con elevado como con bajo riesgo. En población adolescente se cuenta con la ventaja de la familiaridad y uso que tienen de la tecnología, por lo cual programas online y aplicaciones para smartphones tienen el potencial de incrementar el entusiasmo y compromiso con la intervención.

La efectividad de la distribución online de BITs requiere un diseño cuidado incorporando estrategias de diseño de campos como interfaz de usuario, experiencia del usuario, y sistemas persuasivos entre otros. Parks (2014) plantea que con el fin de una adecuada distribución online y abierta al público, las IPP tienen que alcanzar ciertos criterios tanto en su diseño y creación, así como en los tipos de investigación utilizados para evaluar su eficacia. Redzic et al. (2014) han desarrollado un modo de optimizar el diseño de programas online con un sistema de control casi en paralelo y simultáneo. Los programas online de promoción de la salud necesitan ser efectivos y abarcar tanto a una población particular como universal. La programación orgánica (OP en inglés) es un modelo que permite evaluar rápidamente, revisar y redefinir (simultáneamente a su implementación) los programas, para luego redistribuir la versión final en un tiempo acotado. 
Una de las primeras investigaciones empíricas de las intervenciones positivas fue llevada a cabo por internet utilizando una muestra extraída de los visitantes de la página web vinculada al libro La Auténtica Felicidad de Seligman (Schueller \& Parks, 2012; Seligman, Steen, Park, \& Peterson, 2005). También cabe señalar en este sentido la proliferación que tuvo en la red social Facebook el ejercicio de las tres cosas positivas que, a pesar de no tener la aplicación pruebas de eficacia, la diseminación por la red social fomentó su uso (Munson, Lauterbach, Newman \& Resnick, 2010). Otros investigadores también han utilizado distintas plataformas para promover la distribución de IPP. Por ejemplo, Live Happy (Parks et al., 2012) es una aplicación para iPhone. Quienes la usan reportan mejoras en su humor y felicidad, y quienes utilizan más actividades refieren mejoras más significativas en su bienestar.

\section{Clasificación de las Tecnologías Positivas}

En sintonía con lo expresado por Seligman (Conferencia TED, febrero 2004), Riva, Baños, Botella, Wiederhold y Gaggioli (2012) proponen clasificar las tecnologías positivas de acuerdo con los efectos en cada uno de los aspectos de la experiencia personal. En función de la vía del bienestar sobre la que se esté trabajando, es posible identificar tipos de tecnologías positivas: tecnologías hedónicas, tecnologías eudaimónicas y tecnologías sociales o interpersonales (Botella et al., 2012b). En cada nivel hay variables críticas que pueden ser manipuladas y controladas para diseñar y desarrollar adecuadamente TP eficaces.

La tecnología hedónica se centra en el bienestar emocional, desarrollando aplicaciones que fomenten emociones positivas y placenteras o disminuyan estados emocionales negativos en un plazo breve (Botella et al., 2012b). Riva et al. (2012) han puesto énfasis en la importancia de entender cómo las interfaces deberían ser diseñadas para elicitar experiencias emocionales positivas en los usuarios. Para ello, estudiaron distintos componentes y variables a manipular cuando se desee modificar la cualidad afectiva de una experiencia. Según Riva (2012) es posible mediante realidad virtual inducir respuestas emocionales específicas, incluso estados de ánimo positivos. El uso de videos, audios o RV ha demostrado ser beneficioso en la obtención de relajación (Villani, Riva, \& Riva, 2007), siendo importante la sensación de presencia que pudiera alcanzar el usuario (media exposure). Pero se requiere cautela a la hora de proponer estas aplicaciones pues no todas sirven para todos y no todo sujeto está abierto a utilizarlas.

Un ejemplo de estas tecnologías es Positive Technology (tecnología positiva), una aplicación para iPad y iPhone desarrollada en el marco del proyecto de la Unión Europea INTERSTRESS, que se ofrece un entrenamiento en relajación para disminuir los niveles de stress. Cuenta con tres componentes: ejercicios de relajación guiados, con la posibilidad de contar con música y videos apropiados; 
dispositivos de biofeedback que favorecen el aprendizaje del control de sus respuestas fisiológicas mediante la visualización de cambios en su frecuencia cardíaca en un entorno virtual; y el registro y seguimiento de la evolución de su estrés mediante el registro fisiológico y autoinformes (Gaggioli et al., 2014).

Por su parte, Botella et al. (2009a) han desarrollado Mayordomo, un dispositivo que incorpora RV y TICs. Es una plataforma de e-salud diseñada para promover la calidad de vida de las personas mayores. Una de sus aplicaciones es un entorno virtual que representa una caminata por la naturaleza y también contiene una serie de procedimientos de inducción emocional (MIPs, por su sigla en inglés). Su narrativa invita a los participantes a adentrarse en un amplio campo verde donde pueden pasar un agradable día en un entorno vital y colorido o en un contexto pacífico y relajante. A su vez, los sujetos pueden practicar distintos procedimientos de entrenamiento emocional como respiración controlada, estrategias de mindfulness, reminiscencia y otras.

Emotional Parks (Parques Emocionales), también desarrollado por el equipo de Botella (2012b) dentro del Proyecto EMMA, combina MIPs con RV para evocar emociones positivas.

Estas aplicaciones han sido testeadas y han demostrado su capacidad de inducir estados emocionales positivos en los sujetos y disminuir los negativos.

La tecnología eudaimónica consiste en desarrollos que puedan ayudar a los individuos a alcanzar experiencias de compromiso y autorrealización (Riva, 2012). Rogers (2006, citado en Riva et al., 2012) propone un cambio de la computación proactiva a personas proactivas, en el que la tecnología se implementa no para hacer las cosas por las personas sino para involucrarlas más activamente en lo que hacen. En este nivel, se usa la tecnología para apoyar experiencias de flow. Entre los distintos tipos de tecnologías interactivas, los sistemas inmersivos como la RV son los más aptos en la generación de estas experiencias (Riva et al., 2012). Las características de estas tecnologías como fuentes de flow son: oportunidad de acción (con reglas y objetivos), flexibilidad (se pueden crear diversos entornos con distintas tareas y situaciones), y feedback (la RV ofrece retroalimentación de distintas maneras a las conductas del individuo). Para esto es importante el logro de la experiencia de presencia en el entorno virtual.

Riva et al. (2012) sugieren la posibilidad de usar la RV para una variedad de aplicaciones en salud mental positiva basada en la estrategia que definen como transformación de flow. Ésta es la habilidad de acceder, mediante la creación de experiencias transformadoras, a una experiencia óptima inducida por la tecnología y utilizarla para promover nuevos recursos psicológicos y fuentes de participación. El concepto de transformación de flow contiene importantes promesas para la tecnología positiva y sus aplicaciones de RV y dispositivos móviles. Por ejemplo, 
los entornos de realidad virtual pueden ser diseñados para ofrecer situaciones de mayor motivación y desafío, facilitando un compromiso activo de los sujetos con discapacidad en la actividad de juego y rehabilitación. En experiencias de flow, la resiliencia y el bienestar pueden ser promovidos por intervenciones digitalizadas orientadas a una evaluación positiva de sí mismo, al desarrollo personal continuo, a la construcción de un sentido vital, al fomento de relaciones positivas, y al desarrollo de la asertividad y un sentido de autodeterminación (Botella et al., 2012b). Como señala Ryff (1989), la eudaimonía es una combinación de crecimiento personal, control ambiental, propósito, autonomía, autoaceptación y relaciones positivas.

Un ejemplo de este tipo de tecnología podría ser el módulo Book of Life (Libro de la Vida) de la aplicación EARTH que incluye algunos capítulos sobre eventos significativos que han sucedido en la vida de un sujeto así como también planes futuros. Se les ofrece a los usuarios distintos recursos audiovisuales: imágenes, videos, música, textos, y cuando es posible, elementos personalizados. Cada capítulo es diseñado para promover una fortaleza en particular (Baños, Botella, Quero, García Palacios \& Alcañiz, 2011).

También podría considerarse en este grupo algunos juegos serios que promueven experiencias de flow (mediante la regulación del grado de desafío con relación al de las habilidades del sujeto), la tecnología persuasiva para activar el comportamiento deseado o la rehabilitación virtual para aumentar la motivación en diferentes contextos (Riva et al., 2012).

La tecnología social implica el uso de las TICs para promover la integración y conectividad social. Apunta al bienestar social, al self compartido, fomenta la conectividad entre los individuos, grupos y organizaciones, y promueve cambios sociales. Son desarrollos centrados en la promoción de relaciones sociales y en el crecimiento colaborativo y participativo. Desde el punto de vista de las TICs esto se podría lograr por medio de tecnologías como internet y las redes sociales. La conectividad entre miles de personas por intermedio de las distintas redes sociales da lugar a un nuevo y poderoso net-shared-self (self compartido en red). El self trasciende los límites del individualismo; parte de la identidad personal influye en ese nuevo self y la contribución de miles de personas conectadas da forma e influye en la identidad personal de cada uno (Botella et al., 2012b).

Se reveló que el uso de Facebook puede evocar estados psicofisiológicos caracterizados por valencia positiva y elevado arousal (coreflow state, componente esencial del estado de flujo). Esto explica que el éxito de la propagación del uso de las redes sociales puede asociarse con un estado emocional positivo mientras se lo usa (Mauri, Cipresso, Balgera, Villamira, \& Riva, 2011). Un ejemplo de este tipo de tecnología positiva es PosiPost Project (Kanis \& Brinkman, 2008 
citado en Botella et al., 2012b) que incluye varias aplicaciones para compartir emociones positivas y experiencias que promuevan participación y conectividad con otras personas a la distancia.

Actualmente las redes sociales tienen gran influencia en cuestiones sociales, políticas, económicas y demás, aunque en su origen no tenían como objetivo la promoción del bienestar de grupos, sociedades y organizaciones. Aún hay pocos desarrollos de TP en que se integre lo social con el marco de la psicología positiva. Hasta ahora las redes sociales muestran cómo formando parte de algo y compartiendo objetivos significativos puede ser una fuente poderosa de reconocimiento personal y de pertenencia. Incluso el hecho de sentirse uno en el mundo con otros puede ser un importante elemento en la perseverancia hacia las metas propuestas (Botella et al., 2012b).

Es un desafío crear un sentido de conciencia mutuo y recíproco, que es fundamental para sentir la presencia de otro participante y para crear un fuerte sentido de comunidad más allá de la distancia. Riva (2012) propone que una persona está presente en un grupo virtual si es capaz de poner sus propias intenciones y propósitos en práctica, y es capaz de entender las intenciones de otros miembros (social presence, presencia social). Para dar lugar y mantener experiencias sociales óptimas (networked flow) la tecnología tiene que proveer a los grupos virtuales la posibilidad de expresarse a sí mismos y de entender qué está haciendo cada miembro. Gaggioli, Milani, Mazzoni, y Riva (2011) sostienen que estados grupales óptimos se alcanzan cuando el equipo desarrolla una intención grupal en la que las acciones de los individuos y las del grupo están fusionadas, funcionando así como una entidad autónoma y autoorganizada. Es un proceso complicado que se alcanza por intermedio de varios pasos. Morris (2005) ha descripto recientemente cómo las redes sociales y la computación persuasiva pueden ser eficazmente usadas para ayudar a reducir el sentimiento de aislamiento social y depresión en individuos mayores.

Por último, cabe señalar el Crowd-Powered System /Crowdsourcing como IPP (Morris \& Picard, 2014). Esta es una idea un tanto más ambiciosa. Pretende aplicar el concepto y formato de los sistemas de crowdsourcing a la posibilidad de ofrecer IPP. Queda mucho aún por investigar al respecto pero la idea es novedosa y prometedora. Crowdsourcing se refiere a la práctica de derivar un trabajo a una multitud. Esta práctica supone invitar a un gran número de personas, por lo general en internet, a abordar un proyecto de grandes dimensiones de manera conjunta. Permite hacer de manera colectiva, más rápida, mejor y más barata, algo que de otra manera resultaría muy difícil o imposible realizar.

Morris y Picard (2014) proponen que abordajes motorizados por una multitud tienen el potencial de mejorar la adherencia, el compromiso y la eficacia de las 
BITs ofreciendo un intercambio social y, al mismo tiempo, nuevas formas de asistencia. Cuando se gestiona adecuadamente, el crowdsourcing se convierte en una herramienta muy poderosa (e.g. digitalizar millones de documentos; etiquetar millones de imágenes). Explicar el diseño y estructura de estas plataformas excede los límites de este trabajo, sin embargo vale aclarar que cuentan con sofisticados códigos algorítmicos (en su programación) y técnicas de control de la calidad de sus procesos y aportes. Según Morris y Picard (2014) las IPP pueden beneficiarse por estos sistemas crowd-powered (motorizados por una multitud) que pueden ofrecer asistencia a demanda ayudando a reforzar ciertas nociones en el momento en que se lo necesita, intentando responder inteligentemente a la situación particular de una persona.

Lo que ellos plantean es que podrían existir plataformas diseñadas para que cuando una persona necesite asistencia, pueda presentar su situación y que sea la misma red/multitud online quien le provea asesoramiento acerca de cómo encararla, o le promueva el desarrollo del recurso necesario para afrontarla. Muchas veces, los pares podrían ser una buena fuente de asistencia, aunque el apoyo social no siempre está disponible. Las nuevas tecnologías pueden ayudar a satisfacer estas necesidades realizando distintos aportes, por ejemplo, ofreciendo un sistema crowd-powered disponible online permanentemente que pueda responder a la situación particular presentada para un sujeto. Para explorar esto, Morris y Picard (2014) diseñaron un sistema crowdsourcing que ayuda a los usuarios a desarrollar revalorizaciones saludables y positivas de situaciones estresantes. Es necesario realizar más investigaciones y en profundidad con el fin de poder evaluar la conveniencia y eficacia de las respuesta ofrecidas, la propiedad de las mismas, y el sistema de reclutamiento de los agentes de la red. Asimismo, es una cuenta pendiente aún comprobar si este sistema ayuda a que la persona incorpore gradualmente estrategias de revalorización que lleven a prescindir del mismo a futuro. Los abordajes crowd-powered están siendo recientemente desarrollados y son necesarias más investigaciones para comprender cómo podrían usarse eficientemente, qué personas podrían verse beneficiadas, así como cuestiones de programación. Morris y Picard (2014) sostienen que el uso de técnicas crowd-powered para respaldar las IPP es un espacio poco explorado y sugieren que se realicen más investigaciones en un diálogo entre la psicología positiva y el campo de la interacción hombre-computadora.

El futuro de la tecnología positiva deberá tener en cuenta estos desarrollos que permiten interrelacionarse con otros e intercambiar conocimientos, intereses, planes y metas. En ello la contribución de la psicología social también va a ser fundamental (Botella et al., 2012b). Cada uno puede aportar algo que le sirva a otro. El self en red compartido es una entidad a la que todos pueden contribuir y, a la vez, de la que pueden verse beneficiados. 


\section{Conclusiones}

En el presente trabajo se realizó una breve descripción del campo de la Psicología Positiva impulsada por el Dr. Martin Seligman. Asimismo se caracterizó la noción de Tecnología Positiva como ámbito de confluencia de la Psicología Positiva y las TICs. Por último, se procedió a realizar una revisión de algunos desarrollos existentes cuyo objetivo es propiciar intervenciones promotoras del bienestar. Queda aún un vasto campo por investigar y profundizar ya que muchas de estas aplicaciones todavía se encuentran en fases de validación.

Pensando en políticas de promoción de la salud mental, dispensar tratamientos uno a uno tiene poco alcance; es necesario multiplicar el efecto de las intervenciones terapéuticas. La tecnología aumenta la posibilidad de ampliar el impacto de las intervenciones y su duración en el tiempo. En esta línea Kazdin y Blase (2011) proponen usar tecnologías (smartphones, redes sociales, webs), usar medios de comunicación y abrirse a otras disciplinas.

En Argentina es necesaria una mayor difusión y capacitación en la diversidad de recursos existentes con una apertura de los horizontes hacia estilos de comunicación tan incorporados en las nuevas generaciones. Esto abrirá caminos hacia una adaptación de las herramientas ya existentes al contexto sociocultural local, y -aún más desafiante- el desarrollo de nuevos instrumentos atendiendo a las demandas y características regionales. Es necesaria una pronta puesta a punto en el conocimiento de estos recursos para evaluar en conjunto la pertinencia de su implementación en nuestra región y la posibilidad de promover desarrollos locales.

Para finalizar, unas palabras de Seligman:

Creo que con tecnología, entretenimiento y diseño podemos efectivamente aumentar la felicidad humana en el planeta. Y si la tecnología, en las próximas dos décadas, puede aumentar la vida placentera, la vida comprometida y la vida significativa sería más que suficiente. (...) Entonces lo que estaremos haciendo todos en conjunto será suficientemente bueno. (Seligman, Conferencia TED, febrero 2004). 


\section{Referencias}

Baños, R. M., Botella, C., Etchemendy, E., \& Farfallini, L. (2012). EARTH of Wellbeing: a Place to Live Positive Emotions. En B. K. Wiederhold \& G. Riva (Eds.), Annual Review of Cybertherapy and Telemedicine, Advances Technologies in the Behavioral, Social and Neurosciences (pp. 310-313). Amsterdam: IOS Press.

Baños, R.M., Botella, C., Quero, S., García Palacios, A., \& Alcañiz, M. (2011). Engaging Media for Mental Health Applications: the EMMA project. En J. D. Westwood (Ed.), Medicine Mets Virtual Reality 18 (pp. 44-50). Amsterdam: IOS Press.

Baños, R.M., Etchemendy, E., Farfallini, L., García-Palacios, A., Quero, S., \& Botella, C. (2014). EARTH of Well-being System: A Pilot Study of an Information and Communication Technology-Based Positive Psychology Intervention. The Journal of Positive Psychology, 9(6), 482-488.

Botella, C., Etchemendy, E., Castilla, D., Baños, R.M., García Palacios, A., Quero, S., Alcañiz, M., \& Lozano, J.A. (2009a). An ehealth System for Elderly (Butler Project): A Pilot Study on Acceptance and Satisfaction. CyberPsychology \& Behavior, 12, 255-262.

Botella, C., Mira, A., Garcia-Palacios, A., Quero, S., Navarro, M.V., Riera López Del Amo, A., ... Baños, R.M. (2012a). Smiling is Fun: a Coping with Stress and Emotion Regulation Program. En B. K. Wiederhold y G. Riva (Eds.), Annual Review of Cybertherapy and Telemedicine 2012: Advances Technologies (pp. 123-127). Amsterdam: IOS Press.

Botella, C., Quero, S., Serrano, B., Baños, R M, \& García-Palacios, A. (2009b). Avances en los Tratamientos Psicológicos: la Utilización de las Nuevas Tecnologías de la Información y la Comunicación. Anuario de Psicología, 40(2), 155-170.

Botella, C., Riva, G., Gaggioli, A., Wiederhold, B. K., Alcañiz, M., \& Baños, R.M. (2012b). The Present and Future of Positive Technologies. Cyberpsychology, Behavior and Social Networking, 15(2), 1-7.

Bunge, E., López, P., Mandil, J., Gomar M., \& Borgialli, R. (2009). Actitudes de los Terapeutas Argentinos hacia la Incorporación de Nuevas Tecnologías en Psicoterapia. Revista Argentina de Clínica Psicológica, XVIII(3), 209-216. 
Castro Solano, A. (2010). Fundamentos de Psicología Positiva. Buenos Aires: Paidós.

Christensen, H., Griffiths, K. M., \& Farrer, L. (2009). Adherence in Internet Interventions for Anxiety and Depression. Journal of Medical Internet Research, 11(2), e13. doi: 10.2196/jmir.1194

Csikszentmihalyi, M (1997). Fluir (Flow). Una psicología de la Felicidad. Barcelona: Kairós.

Diener, E. (1994). Assessing Subjective Well-being: Progress and Opportunities. Social Indicators Research, 31(2), 103-157.

Drozd, F., Mork, L., Nielsen, B., Raeder, S., \& Bjørkli, C.A. (2014). Better Days -A Randomized Controlled Trial of an Internet-based Positive Psychology Intervention. The Journal of Positive Psychology, 9(5), 377-388.

Fredrickson, B. (1998). What Good are Positive Emotions? Review of General Psychology, 2(3), 300-319.

Gaggioli, A., Cipresso, P., Serino, S., Campanaro, D. M., Pallavicini, F., Wiederhold, B. K., \& Riva, G. (2014). Positive Technology: A Free Mobile Platform for the Self-Management of Psychological Stress. En B. K. Wiederhold \& G. Riva (Eds.), Annual Review of Cybertherapy and Telemedicine 2014 (pp. 25-29). Amsterdam: IOS Press.

Gaggioli, A., Milani, L., Mazzoni, E., \& Riva, G. (2011). Networked flow: A Framework for Understanding the Dynamics of Creative Collaboration in Educational and Training Settings. The Open Education Journal, 1, 41-49.

Gancedo, M. (2008). Historia de la Psicología Positiva. Antecedentes, aportes y proyecciones. En M. M. Casullo (Ed.), Prácticas en Psicología Positiva (pp. 11-38). Buenos Aires: Lugar Editorial.

Kazdin, A. E., \& Blase, S. L. (2011). Rebooting Psychotherapy Research and Practice to Reduce the Burden of Mental Illness. Perspectives on Psychological Science, 6(1), 21-37.

Lyubomirsky, S. (2008). La Ciencia de la Felicidad. Barcelona: Urano.

Mandil, J., Bunge, E., Gomar M., Borgialli, R., \& Labourt, J. (2009). La Implementación de Recursos Tecnológicos en la Clínica con Niños y Adolescentes. Revista Argentina de Clínica Psicológica XVIII(1), 59-68. 
Marks, I., Cavanagh, K., \& Gega, L. (2007). Hands-on Help. Computer-aided Psychotherapy. East Sussex: Psychology Press.

Martínez-Martí, M. L., Avia, M. D., \& Hernández-Lloreda, M. J. (2014). Appreciation of Beauty Training: A Web-Based Intervention. The Journal of Positive Psychology, 9(6), 477-481.

Mauri, M., Cipresso, P., Balgera, A., Villamira, M., \& Riva, G. (2011). Why is Facebook so Successful? Psychophysiological Measures Describe a Core Flow State While Using Facebook. Cyberpsychology, Behavior, and Social Networking, 14(12), 723-731.

Morris, M. E. (2005). Social Networks as Health Feedback Displays. IEEE Internet Computing, 9(5), 29-37.

Morris, R. R., \& Picard, R. (2014). Crowd-powered Positive Psychological Interventions. The Journal of Positive Psychology, 9(6), 509-516.

Munson, S., Lauterbach, D., Newman, M., Resnick, P., (2010). Happier Together: Integrating a Wellness Application Into a Social Network Site. En T. Ploug, P. Hasley, \& H. Oinas-Kukonen (Eds.), Persuasive Technology (pp. 27-39). Berlin: Springer.

Norcross, J. C., Hedges, M., \& Prochaska, J.O. (2002). The face of 2010: A Delphi Poll on the Future of Psychotherapy. Professional Psychology: Research and Practice, 33(3), 316-322.

Norcross, J. C., Pfund, R. A., \& Prochaska, J.O. (2013). Psychotherapy in 2022: a Delphi Poll on its Future. Professional Psychology: Research and Practice, 44(5), 363-370.

Parks, A. C. (2014). A Case for the Advancement of the Design and Study of Online Positive Psychological Interventions. The Journal of Positive Psychology, 9(6), 502-508.

Parks, A. C., Della Porta, M., Pierce, R.S., Zilca, R., \& Lyubomirsky, S. (2012). Pursuing Happiness in Everyday Life: The Characteristics and Behaviors of Online Happiness Seekers. Emotion, 12, 1222-1234.

Peterson, C. (2006). A Primer in Positive Psychology. New York: Oxford University Press.

Redzic, N. M., Taylor, K., Chang, V., Trockel, M., Shorter, A., \& Taylor, C. B. 
(2014). An Internet-based Positive Psychology Program: Strategies to Improve Effectiveness and Engagement. The Journal of Positive Psychology, 9(6), 494-501.

Riva, G. (2012). What is Positive Technology and its Impact on CyberPsychology. En B.K. Wiederhold \& G. Riva (Eds.), Annual Review of Cybertherapy and Telemedicine 2012: Advances Technologies (pp. 37-41). Amsterdam: IOS Press.

Riva, G., Baños, R. M., Botella, C., Wiederhold, B. K., \& Gaggioli, A. (2012). Positive Technology: Using Interactive Technologies to Promote Positive Functioning. Cyberpsychology, Behavior and Social Networking, 15(2), 69-77.

Rose, R. D. (2014). Self-guided Multimedia Stress Management and Resilience Training. The Journal of Positive Psychology, 9(6), 489-493.

Ryff, C. D. (1989). Happiness is Everything, or is it? Explorations on the Meaning of Psychological Well-being. Journal of Personality \& Social Psychology, 57,1069-1081.

Schueller, S. M., \& Parks, A. C. (2012). Disseminating Self-Help: Positive Psychology Exercises in an Online Trial. Journal of medical Internet Research, 14(3), e63. DOI: 10.2196/jmir.1850

Seligman, M. E. P. (2002). La Auténtica Felicidad. Barcelona: Vergara.

Seligman, M. E. P. (2004, feb.). The New Era of Positive Psychology. Conferencias TED-Ed Original. Recuperado de http://www.ted.com/talks/ martin_seligman_on_the_state_of_psychology?language=es

Seligman, M. E. P. (2011). La Vida que Florece. Barcelona: Ediciones B.

Seligman, M. E. P. \& Csikszentmihalyi, M. (2000). Positive Psychology. An Introduction. American Psychologist, 55(1), 5-14.

Seligman, M. E. P., Steen, T., Park, N., \& Peterson, C. (2005). Positive Psychology Progress: Empirical Validation of Interventions. American Psychologist, 60(5), 410-421.

Villani, D., Riva, F., \& Riva, G. (2007). New Technologies for Relaxation: The Role of Presence. International Journal of Stress Management, 14, 260-274.

Wiederhold, B. (2012). A Brief Review of Positive Technology in Europe and the USA. En B. K. Wiederhold \& G. Riva (Eds.), Annual Review of Cybertherapy and Telemedicine 2012: Advances Technologies (pp. 46-50). Amsterdam: IOS Press. 
